\title{
Different patterns of integral membrane protein localization during cell division in Bacillus subtilis
}

\begin{abstract}
Correspondence
P. J. Lewis

Peter.Lewis@newcastle.edu.au
\end{abstract}

Received 14 September 2007

Revised 14 October 2007

Accepted 18 October 2007

\author{
D. H. Meredith, M. Plank and P. J. Lewis
}

School of Environmental and Life Sciences, University of Newcastle, Callaghan, NSW 2308, Australia

\begin{abstract}
Cell division in rod-shaped bacteria nearly always occurs exactly at mid-cell and is dependent on the formation of the cytokinetic FtsZ ring and its associated division proteins. Many thousands of copies of division, or septum-specific proteins assemble at this site and may lead to the exclusion of other integral membrane proteins that are normally able to diffuse freely throughout the cytoplasmic membrane. In this study we have investigated the localization of a series of integral membrane proteins in Bacillus subtilis and we show that the recruitment of division and septum-specific proteins does not necessarily preclude the diffusion of other integral membrane proteins. However, some proteins, namely ATP synthase and succinate dehydrogenase, are reduced/absent from the mid-cell region at the onset of cell division, which may reflect an association with lipid domains rich in phosphatidylglycerol that are thought to be present at diminished levels at sites of cell division.
\end{abstract}

\section{INTRODUCTION}

Cell division in rod-shaped bacteria is an intensively studied process involving the coordinated action of a suite of proteins that assemble at the mid-cell (Errington et al., 2003). Although not all cell-division proteins are conserved, the tubulin homologue FtsZ is present in virtually all bacteria, some chloroplasts and mitochondria from ancient eukaryotes (Gilson \& Beech, 2001). FtsZ selfpolymerizes to form a ring at mid-cell, driving the invagination of membrane and cell wall throughout cell division (Errington et al., 2003). It is also the first known protein to assemble at new division sites (Errington et al., 2003) and so represents an excellent early marker for the location of cell division. Although it is a cytoplasmic protein, FtsZ assembles as a polymer adjacent to the inside surface of the cytoplasmic membrane in order to lead the coordinated assembly of the division complex comprising a series of cytoplasmic and integral membrane proteins (Errington et al., 2003). FtsZ placement is dependent on the action of the Min and Noc (nucleoid occlusion) systems, which ensure that division occurs at mid-cell and will not bisect a replicating chromosome (Errington et al., 2003; Wu \& Errington, 2004; Rothfield et al., 2005).

The mature Bacillus subtilis division complex (the divisome) comprises eight different proteins (Errington et al., 2003; Hamoen et al., 2006; Harry et al., 2006) and many thousands of protein molecules. For example, it is estimated that there are around 50000 molecules of

Abbreviations: CFP, cyan fluorescent protein; CL, cardiolipin; CR, cleared region; GFP, green fluorescent protein; PE, phosphatidylethanolamine; PG, phosphatidylglycerol; YFP, yellow fluorescent protein.
DivIC per cell during exponential growth (Katis et al., 1997). All of these proteins have been shown to localize to the sites of cell division, and are integral membrane proteins, apart from FtsZ and FtsA (Errington et al., 2003; Hamoen et al., 2006; Harry et al., 2006).

The cytoplasmic membrane is also thought to be important in cell division (see Mileykovskaya \& Dowhan, 2005; Matsumoto et al., 2006 for reviews). The major phospholipids found in B. subtilis are phosphatidylglycerol (PG) and phosphatidylethanolamine (PE), although the minor component cardiolipin (CL) is also considered an important membrane lipid due to its packing and non-bilayer formation properties (de Mendoza et al., 2002; Kawai et al., 2004; Matsumoto et al., 2006). Sites of cell division become enriched in CL and PE; this is thought to be related to their ability to form non-bilayer structures that would be important in septation events (Matsumoto et al., 2006). There is now ample evidence that the mid-cell and polar regions have a different lipid profile to the longitudinal regions of the membrane (Binenbaum et al., 1999; Fishov \& Woldringh, 1999; Mileykovskaya \& Dowhan, 2000; Kawai et al., 2004; Nishibori et al., 2005) and so proteins with specific lipid preferences may show altered localization during cell division. It is also possible that due to the large amount of division-specific protein at mid-cell, other integral membrane proteins could be physically excluded from the mid-cell region during division due to crowding effects.

We have investigated the localization of integral membrane proteins in B. subtilis and found that some, but not all, are transiently diminished/absent from mid-cell around the time of FtsZ-ring assembly. At this stage of the cell cycle, 
the amount of known division-specific integral membrane protein present at mid-cell would be low. All the proteins investigated were present at mid-cell during the later stages of division when the level of division-specific proteins was at its highest. The specific reduction of proteins from the mid-cell region was also shown to be dependent on division ring formation and not on other processes such as DNA replication, which is also known to occur around mid-cell (Lemon \& Grossman, 1998; Migocki et al., 2004). Consistent with studies on the localization of specific lipids, we interpret our data as suggesting that integral membrane proteins migrate into division septa at different rates dependent on their local lipid environment.

\section{METHODS}

Bacterial strains and media. All cloning was carried out in Escherichia coli DH5 $\alpha$ (Gibco-BRL). B. subtilis strains used and constructed in this work are listed in Table 1. Transformation of $B$. subtilis was carried out by the method of Anagnostopoulous \& Spizizen (1961), as modified by Jenkinson (1983). Transformants were selected on nutrient agar containing the appropriate antibiotic (chloramphenicol $5 \mu \mathrm{g} \mathrm{ml}^{-1}$, spectinomycin $50 \mu \mathrm{g} \mathrm{ml}^{-1}$ ). When used to control expression of genes, xylose and IPTG were used at $0.5 \%$ $(\mathrm{w} / \mathrm{v})$ and $0.5 \mathrm{mM}$, respectively, unless otherwise stated. For microscopy and cell measurement experiments, all strains were grown at $37^{\circ} \mathrm{C}$ in $\mathrm{CH}$ medium as described previously (Davies et al., 2005). FtsZ-depletion experiments were performed by growing cells in $\mathrm{CH}+0.5 \mathrm{mM}$ IPTG to an $\mathrm{OD}_{600}$ of 0.3 , pelleting the cells, then resuspending them in pre-warmed $\mathrm{CH}$ medium lacking IPTG. This point was taken as $t_{0}$ and further samples were taken at the appropriate times as indicated in the text.

DNA manipulations. p16.7 was amplified from BS125 chromosomal DNA (Table 1) using the primers Forward 5'-AACAAGCTGGCCTAGGATGGAAGCTATTTTGATG- $3^{\prime}$ and Reverse $5^{\prime}$-CAACAAGCTTTTTCAATGACCCCCGATATAGT-3'. The resulting product was digested with AvrII and HindIII (sites underlined in primer sequences) and inserted into similarly cut pSG1192 to generate pNG510 (Table 1). secDF was amplified from strain 168 chromosomal DNA using the primers Forward 5'-GAGGATACTCGAGATGAAAAAAGGACG-3' and Reverse 5'-GCCTGATATGAATTCTTGCGCCGA-3'. The resulting product was digested with XhoI and EcoRI and inserted into similarly cut pSG1729 to give pNG534 (Table 1). The integrity of inserts was confirmed by DNA sequencing. These plasmids were used to transform competent $B$. subtilis strains as listed in Table 1.

Microscopy, image acquisition and analysis. Microscopy was performed as detailed previously (Davies et al., 2005) by mounting cells on $1.2 \%(\mathrm{w} / \mathrm{v})$ agarose pads, followed by imaging with a Zeiss Axioscop 2 fluorescence microscope fitted with a Photometrics Quantix 1401E cooled CCD camera. Cyan fluorescent protein (CFP) fluorescence was visualized with filter set $31044 \mathrm{v} 2$, green fluorescent protein (GFP) with set 41018, and yellow fluorescent protein (YFP) with set 41029 (Chroma Technology). Image processing and analysis was performed as described by Johnson et al. (2004) using MetaMorph v6.1.02 software (Molecular Devices). When performing linescans, a line was drawn right around the cell periphery starting at

Table 1. Strains and plasmids used in this study

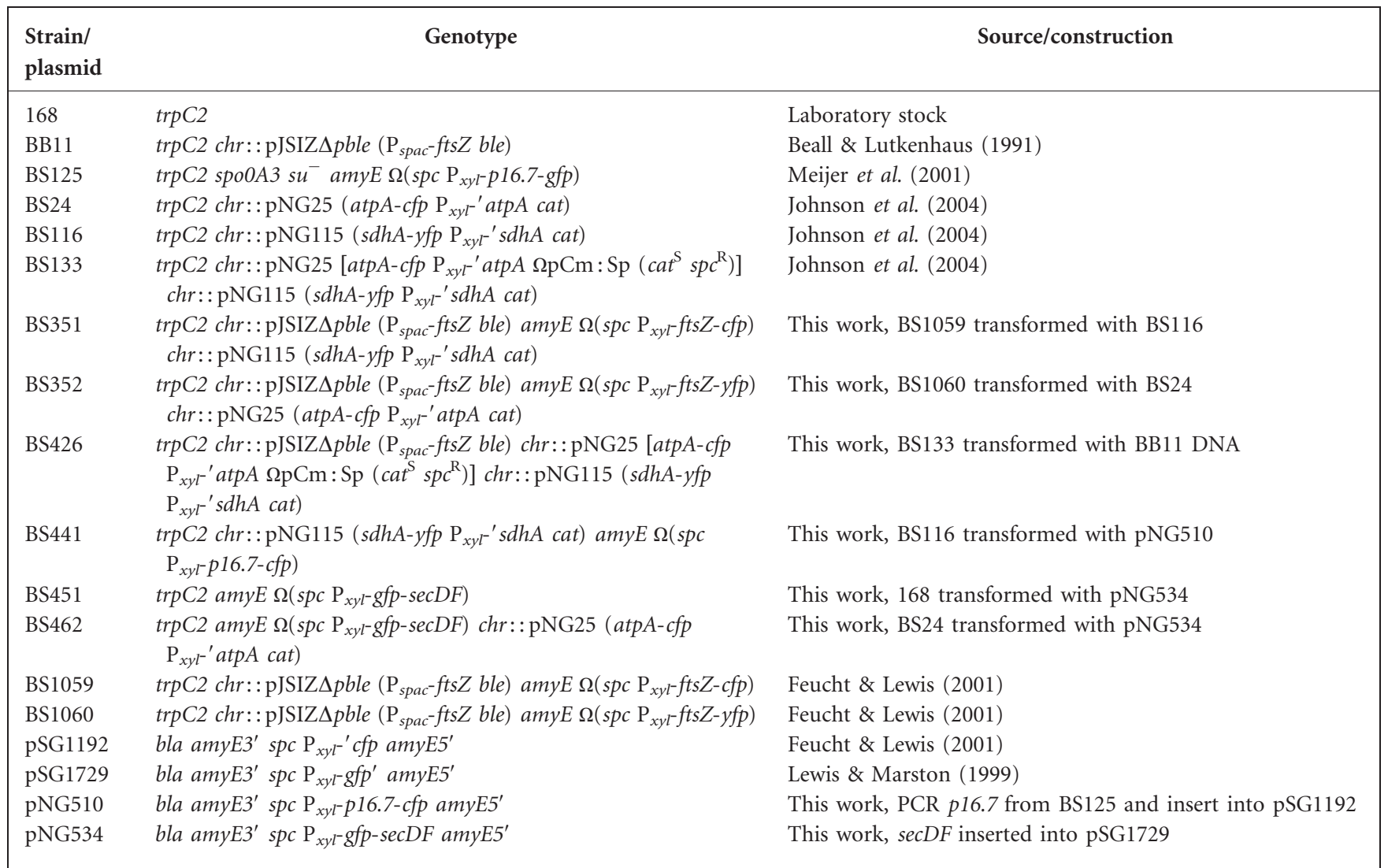


one of the cell poles. In the resulting intensity profile, the mid-cell sites would therefore correspond approximately to the one-quarter and three-quarter points on the $x$-axes. Final images were prepared for publication using Adobe Photoshop.

\section{RESULTS}

\section{Colocalization of integral membrane proteins}

In previous studies we investigated the distribution of succinate dehydrogenase and ATP synthase complexes using fluorescent protein fusions to the AtpA and SdhA subunits, respectively, which showed that both complexes were distributed around the cytoplasmic membrane (Johnson et al., 2004). The expression of both fluorescent fusions was driven by the activity of the wild-type promoters, and polar effects of insertion of the fusions into the chromosome were avoided through expression of downstream genes from the xylose-inducible promoter (see Johnson et al., 2004 and Fig. 1A). The pattern of localization for both SdhA and AtpA fusions was heterogeneous, with multiple small regions of fluorescence of varying levels of intensity (see Johnson et al., 2004). Due to this highly varied level of fluorescence around the cytoplasmic membrane, no obvious pattern of low signal at mid-cell was apparent in either of the single-labelled strains. However, we subsequently noticed that a proportion of the cells in the atpA-cfp sdhA-yfp dual-labelled strain BS133 (Table 1) showed much lower levels of signal at the mid-cell site (Fig. 1B). As is clear in the linescan of the cell perimeter shown in Fig. 1(D), the distribution of each fusion was heterogeneous as marked by a series of peaks and troughs, but at the mid-cell region (light and dark grey arrows, Fig. 1C, D) there was a coincident drop in signal intensity for both fusions. These areas, corresponding to simultaneous dips in the signal of dual fluorescent labels at mid-cell, were subsequently termed cleared regions (CRs) and were found to be present in $9.4 \%$ of exponentially growing cells (Table 2 ). It should be noted that whilst there are many peaks and troughs for the ATP synthase and succinate dehydrogenase signals, the coincident drops in signal representing CRs only occurred at mid-cell (see arrowed cells, Fig. 1B, C).

In order to determine how widespread CRs were, we examined the localization of some additional integral membrane proteins. Strain BS441 contains a xyloseinducible $p 16.7-c f p$ fusion along with the $s d h A-y f p$ fusion (Fig. 2A; Table 1). p16.7 is an integral membrane protein from the B. subtilis phage $\phi 29$ that is involved in phage DNA replication, and has previously been shown to localize around the cell membrane when ectopically expressed (Meijer et al., 2001; Johnson et al., 2004). This protein plays no role in the metabolism of uninfected B. subtilis and so was used to determine if the appearance of CRs was a non-specific effect on integral membrane proteins at the onset of cell division. Strain BS462 containing an Nterminal $g f p$ fusion to $s e c D F$ along with the atpA-cfp fusion
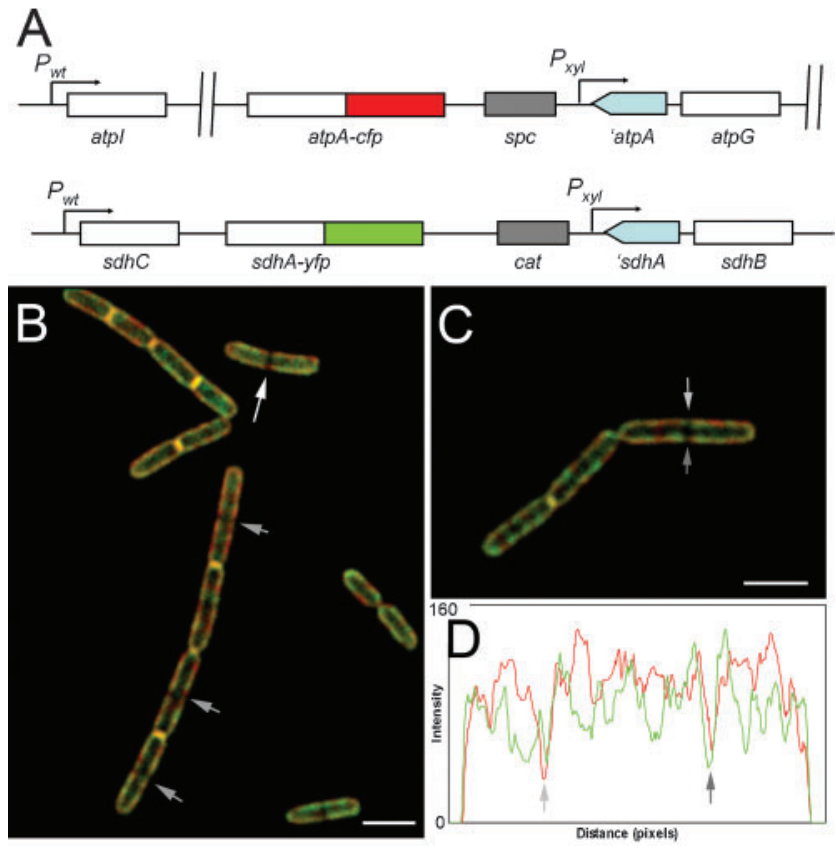

Fig. 1. Colocalization of the integral membrane proteins ATP synthase and succinate dehydrogenase. (A) Schematic of the chromosomal construction of strain BS133, carrying an atpA-cfp and $s d h A-y f p$ fusion, that was produced by Johnson et al. (2004). Transcription of each of the fluorescent protein fusions was driven by the wild-type atp or sdh promoters, whilst expression of genes downstream from the sites of insertion was controlled by the xylose-inducible promoter $\mathrm{P}_{x y l}(\mathrm{~B}, \mathrm{C})$ Overlays of AtpA-CFP (red) and SdhA-YFP (green) fluorescent signals in cells of strain BS133 growing exponentially in $\mathrm{CH}$ medium. The white arrow in (B) indicates a cell in which there is a well-defined mid-cell region of low red and green fluorescent signals. The grey arrows indicate the mid-cell regions in a chain of cells where there is a lowering of fluorescent signal at mid-cell, but to a lesser degree than for the cell indicated with the white arrow. A linescan of the arrowed cell in (C) is displayed in (D). The light and dark grey arrows on the micrograph indicate regions in the linescan where there is a reciprocal drop in fluorescent signal from both fusions at the midcell point. Scale bars, $2 \mu \mathrm{m}$.

was also constructed (Fig. 2E; Table 1). SecDF, as part of the Sec secretion complex, is involved in protein secretion (Dalbey \& Chen, 2004) and so is not directly associated with the same metabolic activities as ATP synthase and succinate dehydrogenase. Therefore, this strain would inform us whether CRs were a phenomenon that occurred with all $B$. subtilis integral membrane proteins, or just a subset.

We were unable to obtain a fluorescent YFP fusion to SecDF and so the dual-labelled BS462 strain contained a CFP and GFP fusion. The level of spectral crossover of GFP signals into CFP channels and vice versa was determined with single-labelled strains BS24 and BS451 (Table 1) and shown to be insignificant (not shown). Previous work has 
Table 2. Frequency of mid-cell clearing in strains carrying fluorescent protein fusions to integral membrane protein complexes

\begin{tabular}{|lccccc|}
\hline \multirow{2}{*}{$\begin{array}{l}\text { Time } \\
(\min )^{*}\end{array}$} & \multicolumn{2}{c}{ BS133 } & & \multicolumn{2}{c|}{ BS426 } \\
\cline { 2 - 3 } \cline { 5 - 5 } & $\begin{array}{c}\text { Mean cell } \\
\text { length }(\boldsymbol{\mu})\end{array}$ & $\begin{array}{c}\text { Frequency of } \\
\text { CRs }(\%) \dagger\end{array}$ & $\begin{array}{c}\text { Mean cell } \\
\text { length }(\boldsymbol{\mu m})\end{array}$ & $\begin{array}{c}\text { Frequency } \\
\text { of CRs }(\%) \dagger\end{array}$ \\
\hline 0 & 3.3 & 9.4 & & 4.3 & 6.3 \\
45 & & & 6.0 & 1.9 \\
90 & & & 9.1 & 0 \\
150 & & & 17.2 & 0 \\
180 & & & 23.3 & 0 \\
\hline
\end{tabular}

${ }^{*}$ Number of minutes following removal of IPTG from the medium (for strain BS426 only).

†CRs, 'cleared regions', corresponding to coincident drops in fluorescence on opposite sides at regular intervals along the length of the cell. At least 250 cells were counted for each sample.

also shown that CFP and GFP fusions can be unambiguously localized with little or no signal crossover (Davies \& Lewis, 2003) and so strain BS462 was used to colocalize SecDF and ATP synthase.
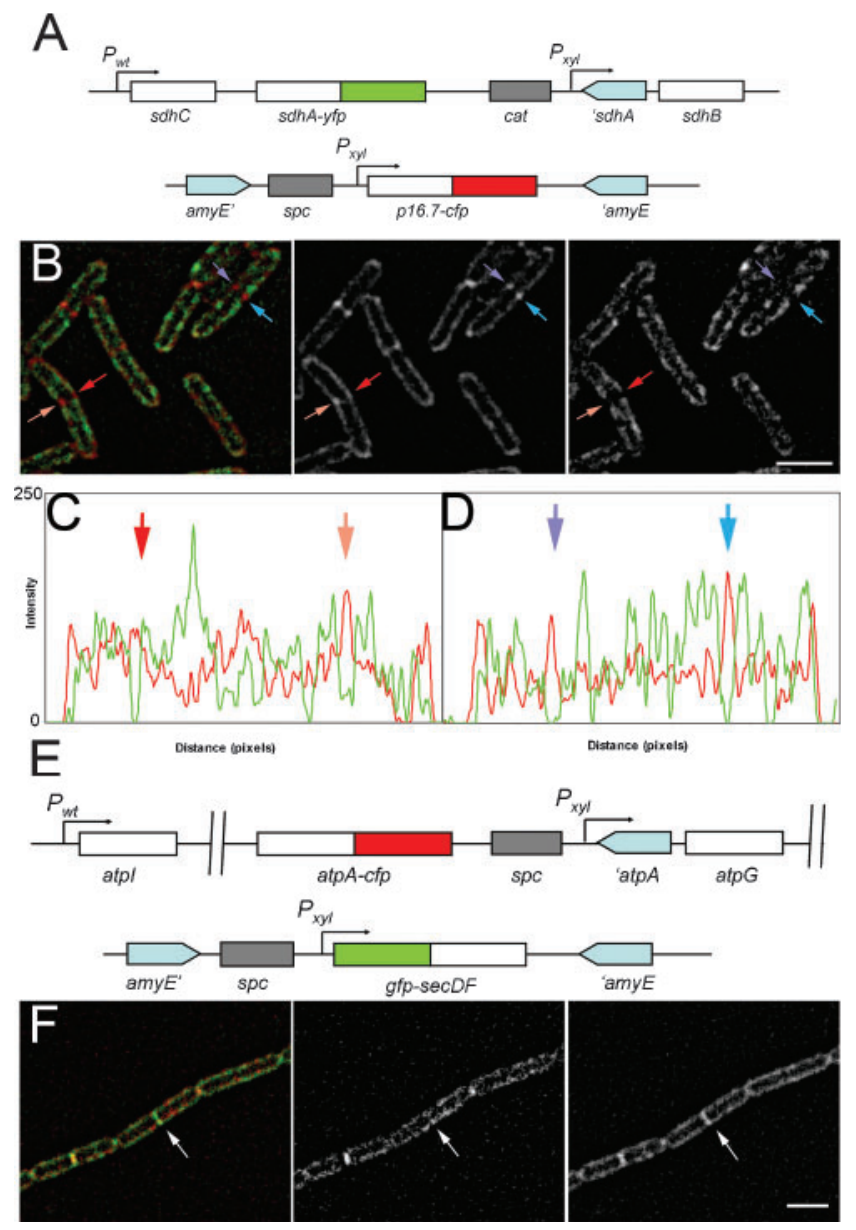

Fig. 2(B, C, D) shows the results of examination of strain BS441. Surprisingly, there was no evidence that p16.7 formed CRs, and instead a p16.7 signal was seen to concentrate at division sites concomitant with a drop in the SdhA-YFP signal (arrows, Fig. 2B, D). At early stages of cell division around the time that septal invagination was just beginning, p16.7-CFP signals appeared as slight concentrations of signal at mid-cell (red arrows, Fig. 2B, C), that became more intense as the divisome matured (blue arrows, Fig. 2B, D).

Similarly, no evidence for CRs could be found with strain BS462, and as with p16.7, SecDF appeared to localize to septal regions/membrane ingrowths at an earlier stage of cell division than ATP synthase (Fig. 2F, arrows). Thus, the phenomenon of CRs appears to be restricted to a subset of integral membrane proteins currently comprising the ATP synthase and succinate dehydrogenase complexes.

\section{Cleared regions occur early in the division cycle}

The occurrence of CRs only at mid-cell sites indicated that they could be connected to the cell division cycle. In order to investigate the role of cell division in the clearing of integral membrane proteins from the mid-cell site, further strains were constructed to contain dual fluorescent protein fusions to either $f t s Z$ and $s d h A$ (BS351; Table 1, Fig. 3A), or ftsZ and atpA (BS352; Table 1). In these strains expression of wild-type FtsZ was dependent on the

Fig. 2. Localization of integral membrane proteins p16.7 ( $\phi 29)$ and SecDF with ATP synthase and succinate dehydrogenase. (A) Schematic of the chromosomal construction of strain BS441 carrying an $s d h A-y f p$ and a $p 16.7-c f p$ fusion. Transcription of the $s d h A-y f p$ fusion was driven by the wild-type $s d h$ promoter, whilst expression of $p 16.7-c f p$ was controlled by the xylose-inducible promoter $\mathrm{P}_{x y /}$. (B-D) Colocalization of SdhA-YFP and p16.7-CFP in strain BS441. In the left-hand micrograph in (B), the SdhA-YFP signal is pseudocoloured green and the p16.7-CFP signal, red. The p16.7-CFP signal is shown in the middle micrograph, and the SdhA-YFP signal in the right-hand micrograph. Cells containing regions corresponding to accumulations of $\mathrm{p} 16.7-\mathrm{CFP}$ signal and SdhA-YFP CRs are arrowed either red or blue (see text for details). (C) The linescan around the perimeter of the red-arrowed cell with the dark and light red arrows corresponding to the regions indicated in the cell in (B). (D) The linescan around the perimeter of the blue-arrowed cell with the dark blue and cyan arrows corresponding to the regions indicated in the cell in (B). (E) Schematic of the chromosomal construction of strain BS462 carrying an atpA-cfp and $g f p-s e c D F$ fusion. Transcription of the atpA-cfp fusion was driven by the wild-type atp promoter, whilst expression of gfp-secDF was controlled by the xylose-inducible promoter $\mathrm{P}_{x y /}$. In the left-hand micrograph in (F), the GFP-SecDF signal is pseudocoloured green and AtpA-CFP signal, red. The AtpA-CFP signal is shown in the middle micrograph, and the GFPSecDF signal in the right-hand micrograph. The arrow in all micrographs corresponds to a mid-cell region in which GFPSecDF has migrated into the new septum, but Atp-CFP (ATP synthase) is absent. Scale bars, $2 \mu \mathrm{m}$. 

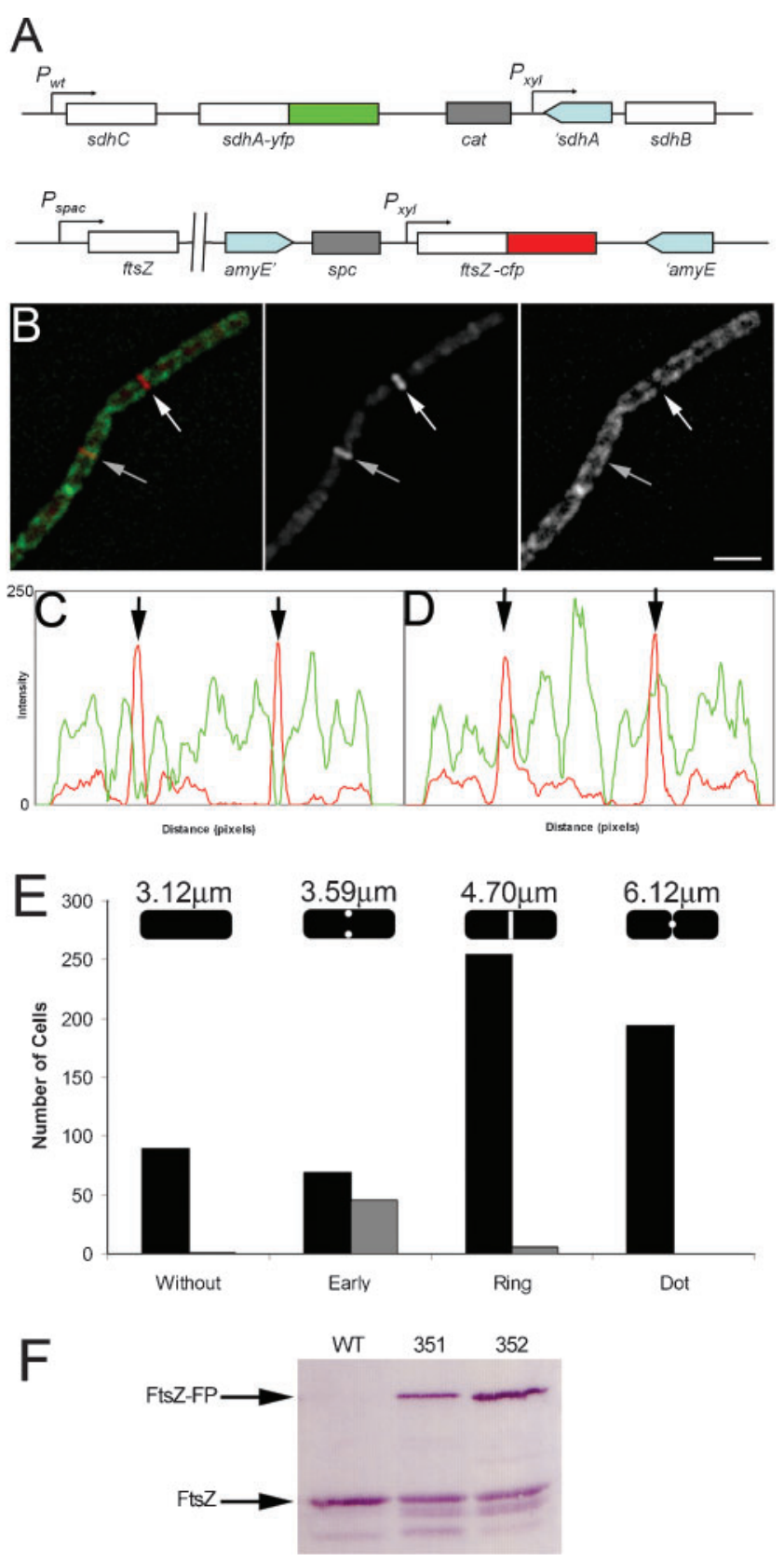

presence of IPTG, whereas expression of fluorescently tagged FtsZ was dependent on the presence of xylose. Production of wild-type FtsZ was necessary in these strains as the fluorescently tagged protein is not fully functional (Feucht \& Lewis, 2001). The strains also contained a fusion to either $s d h A$ or atpA, both of which lie within operons and require the presence of xylose for the expression of genes within the operons downstream of the site of insertion of the fluorescent protein fusion (see Johnson et al., 2004 and Fig. 3A). Titration of strains with IPTG and xylose indicated that an approximately wild-type cell-length distribution, along with acceptable levels of fluorescence, could be obtained with $0.1 \mathrm{mM}$ IPTG and
Fig. 3. Protein-free mid-cell regions correspond to the early stages of cell division. (A) Schematic of the chromosomal construction of strain BS351, carrying an sdhA-yfp and ftsZ-cfp fusion. Transcription of the $s d h A-y f p$ fusion was driven by the wildtype $s d h$ promoter, whilst expression of genes downstream from the sites of insertion, and the ftsZ-cfp fusion was controlled by the xylose-inducible promoter $\mathrm{P}_{x y \mid}$. Expression of wild-type FtsZ was under the control of the IPTG-inducible $P_{\text {spac }}$ promoter. In the lefthand micrograph in (B), SdhA-YFP fluorescence has been pseudocoloured green and the FtsZ-CFP signal, red. The FtsZCFP signal is shown in the middle micrograph, and the SdhA-YFP signal in the right-hand micrograph. The white arrow indicates a cell containing a coincident Z-ring and $C R$, whilst the grey arrow indicates a cell containing a Z-ring, but no CR. Scale bar, $2 \mu \mathrm{m}$. (C) Linescan around the perimeter of the white-arrowed cell, (D) linescan of the grey-arrowed cell from (B); the black arrows correspond to the mid-cell sites (and FtsZ peaks). (E) Bar chart illustrating the distribution of cells at different stages of the division cycle and the correlation of CRs with the morphology of FtsZ division rings for strain BS351. The cartoons in $(E)$ indicate the appearance of FtsZ rings at different stages of the division cycle; mean cell sizes are shown above each cartoon. Classes of cells are as follows: without, no FtsZ ring apparent; early, the FtsZ ring appears as two dots on opposite sides of the mid-cell representing the early stages of cell division when other division proteins are still being recruited to the mid-cell site; ring, a mature FtsZ ring is present which appears as a band across the middle of the cell and represents a cell undergoing the initial stages of division; dot, represents the appearance of the FtsZ ring at later stages of the division cycle when invagination of the cell walls is clearly visible by phase-contrast microscopy. Black columns represent cells in which no CR could be seen. Grey columns represent cells in which CRs could be observed. (F) Western blot of whole-cell extracts immunoblotted with anti-FtsZ antibody. Equivalent amounts of protein were loaded in each lane. WT corresponds to strain 168 (wild-type), 351 and 352 to the dual-labelled strains containing FtsZ fluorescent protein fusions (BS351 and BS352) grown under the conditions detailed in the text.

$0.2 \%(\mathrm{w} / \mathrm{v})$ xylose. Western blots indicated that the total level of FtsZ (wild-type plus fusion protein) was slightly higher than the wild-type level of FtsZ in the parent strain 168 (Fig. 3F). The mean cell length of BS351 was $4.03 \pm 0.96 \mu \mathrm{m}$ compared to $3.6 \mu \mathrm{m}$ obtained for the wild-type strain 168 grown in identical conditions by Sharpe et al. (1998). The small difference in mean cell length may be due to the combined levels of FtsZ and FtsZYFP being suboptimal in this strain.

Cultures of strains BS351 (sdhA, ftsZ fusions; Table 1) and BS352 (atpA, fts $Z$ fusions; Table 1) were grown and examined microscopically. An image of a chain of cells from strain BS351 along with linescans around the perimeter of the marked cells is shown in Fig. 3(B, C, D). For both strains, regions of low membrane signal corresponded with FtsZ division rings (white arrow Fig. 3B, and black arrows Fig. 3C). However, CRs were not present in all cells with visible FtsZ rings (grey arrow Fig. 3B, and black arrows Fig. 3D). In these cells, the SdhA signal can be 
clearly seen to overlap the FtsZ signal at mid-cell. These results suggested that CRs may occur at a specific stage of the division cycle and so a detailed analysis of CR appearance with FtsZ rings and cell length was conducted (Fig. 3E). Cells were classified as having no detectable FtsZ ring (Fig. 3E) ('without', mean cell length $3.12 \pm 0.52 \mu \mathrm{m}$ ), an early-stage division ring that appeared as two dots on opposite sides of mid-cell (morphologies first characterized for DivIC by Katis et al. (1997); 'early', $3.59 \pm 0.49 \mu \mathrm{m}$ ), a mature division ring ('ring', $4.70 \pm 0.74 \mu \mathrm{m}$ ), or to be in the late stages of cell division ('dot', $6.12 \pm 0.42 \mu \mathrm{m}$ ). Both the sdhA fts $Z$ and atpA fts $Z$ dual-labelled strains had similar proportions of each class, and similar proportions of cells in which CRs were observed and so results are only shown for the $s d h A$ ftsZ strain BS351 in Fig. 3(E). The majority of cells containing CRs were in the early stage of cell division (Fig. 3E, grey columns) indicating that formation of CRs occurred prior to the full assembly of the division complex, which occurs at the 'ring' stage of the division cycle. This effect was not observed in all the cells. It may be a specific response to a very short-lived event in the division cycle not fully resolved in our analysis, or the lowering of midcell signal may have been much less in some cells than others. Repeated attempts were made to perform timelapse imaging on strains BS351 and BS352 to definitively establish the timing of CR formation with cell division, but due to the rapid photobleaching of the FtsZ fusion we were unable to obtain usable data.

The division complex represents a significant number of protein molecules and it is possible to envisage a situation whereby the assembly of a large number of proteins involved in synthesis of the division septum might exclude other integral membrane proteins, so creating the lowering of signal we observed. However, since CRs occurred at an earlier stage of the division cycle than the full assembly of the division complex, and were not observed for all integral membrane protein fusions, there must be another explanation.

\section{Mid-cell clearing is not due to DNA replication or chromosome segregation cycles}

Due to the overlapping nature of the bacterial cell cycle, DNA replication, chromosome segregation and cell division may all be ongoing in the same cell simultaneously. DNA replication occurs in replication factories positioned approximately around the mid-cell point (Lemon \& Grossman, 1998; Migocki et al., 2004), and it was possible that CRs coincided with some aspect of the replication or segregation cycles. To investigate this possibility, strain BS426 was constructed (Fig. 4A; Table 1), in which the expression of $f t s Z$ was dependent on induction with IPTG. Thus, by removing IPTG from the medium, FtsZ levels in the cells would drop, resulting in the formation of filaments. In filamentous cells, it is well established that DNA replication and segregation occur normally until the cells grow too large and lyse (e.g. see
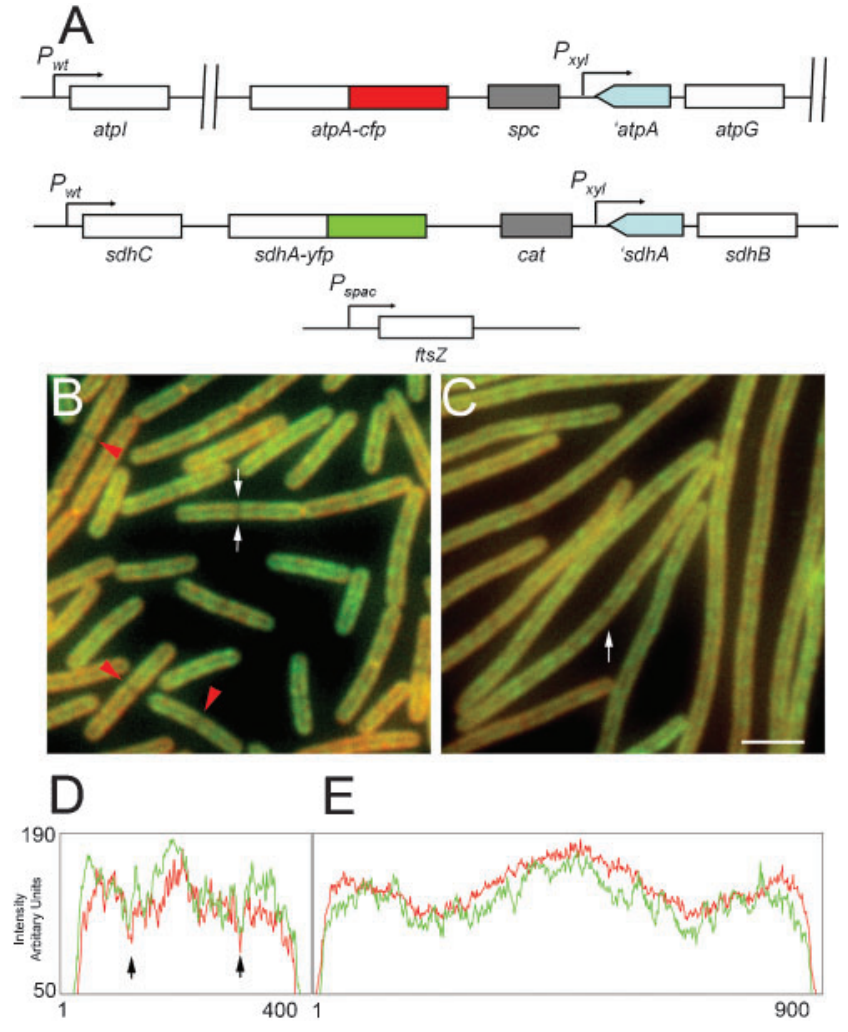

Fig. 4. CRs are dependent on cell division. (A) Schematic of the chromosomal construction of strain BS426, carrying sdhA-yfp and atpA-cfp fluorescent protein fusions along with an inducible copy of ftsZ. Transcription of the $s d h A-y f p$ and atpA-cfp fusions was driven by their wild-type promoters, whilst expression of genes downstream from the sites of insertion was controlled by the xylose-inducible promoter $\mathrm{P}_{x y l}$. Expression of wild-type Fts $\mathrm{Z}$ was under the control of the IPTG-inducible $P_{\text {spac }}$ promoter. (B) $A$ typical field of cells of strain BS426 grown in the presence of 0.5 mM IPTG, with AtpA-CFP pseudocoloured red and SdhA-YFP green. White arrows mark the CRs in the cell for which a linescan was made. Red arrowheads indicate additional cells in which CRs could be seen. (C) An image of the same strain 150 min after removal of IPTG. The cell marked with the arrow was used to obtain a linescan. Scale bar $2 \mu \mathrm{m}$. (D, E) Linescans around the perimeter of the white-arrowed cells from (B) and (C), respectively, with the black arrows corresponding to the position of CRs.

Fig. 5 in Wu et al., 1995; Lewis et al., 2000). So, if mid-cell clearing was dependent on aspects of the replication and segregation cycles independent of Z-ring formation, CRs should still appear at regular intervals along filaments in this strain.

Strain BS426 was grown in $\mathrm{CH}$ medium containing $0.5 \mathrm{mM}$ IPTG to $\mathrm{OD}_{600} 0.3$ prior to resuspension in IPTG-free medium (see Methods). Cells were then collected for analysis at various time points following resuspension, and the frequency of CRs and cell length measurements were determined. The results were compared against the data for strain BS133, which is the 
parent strain of BS426 with wild-type fts $Z$ expression (Table 2). Immediately following resuspension $\left(t_{0}\right)$ cells of strain BS426 had a mean cell length of $4.3 \mu \mathrm{m}$ which is slightly longer than that of the parent strain BS133 (mean $3.3 \mu \mathrm{m}$ ). Titration of FtsZ levels in strain BS426 was attempted to achieve a mean cell length closer to that of BS133, but increasing or decreasing IPTG levels (0.1$1 \mathrm{mM}$ ) did not result in improved mean cell lengths, and the most homogeneous cell-length distributions were obtained using $0.5 \mathrm{mM}$ (not shown).

The frequency of CRs was slightly lower in BS426 compared to BS133 $(6.3 \%$ and $9.4 \%$, respectively; Table 2). In addition, the mean cell length for cells containing CRs was determined as well as the relative position of the CR along the long axis of the cell, where a measurement of 0.5 indicates the mid-point. The mean length at which CRs appeared in BS133 was $4.2 \pm 0.49 \mu \mathrm{m}$ with a relative position of $0.52 \pm 0.03$, and in BS426 was $5.6 \pm 0.9 \mu \mathrm{m}$ with a relative position of $0.51 \pm 0.04$. Therefore, although cells of strain BS426 were slightly longer than the parent strain, CRs still formed in the same relative position, indicating that the mechanism affecting this phenomenon was operating normally in these cells.

Following resuspension in IPTG-free medium, strain BS426 continued to grow as indicated by the exponential increase in mean cell length over the $180 \mathrm{~min}$ time-course when cells increased in mean length from 4.3 to $23.3 \mu \mathrm{m}$ (Table 2). As the mean cell length increased, the frequency of CRs (classified for these cells as concomitant drops in fluorescent signals at regular intervals along filaments rather than a single region at mid-cell) decreased so that by 90 min (mean cell length $9.1 \mu \mathrm{m}$ ) no more could be detected at mid-cell or any other position along the cell length, indicating the dependence of $\mathrm{CR}$ formation on $\mathrm{fts} Z$ expression and that this phenomenon was not dependent on DNA replication or segregation.

\section{DISCUSSION}

In this work we have shown that certain integral membrane proteins are not observed at the mid-cell membrane region during the early stages of cell division. This process is not ubiquitous, but restricted to a subset of proteins, currently comprising the ATP synthase and succinate dehydrogenase complexes. Additional proteins tested were not absent from mid-cell at any stage of the cell cycle. The phage protein p16.7 is small $(16.7 \mathrm{kDa})$ and so the presence of this protein at mid-cell could be attributed to a higher diffusion rate within the membrane compared to the larger ATP synthase and succinate dehydrogenase complexes. Also, since p16.7 is not a component of the normal cellular proteome, it may not respond to cytoplasmic signals in the same way as normal cellular proteins. However, no evidence of CRs was observed using the SecDF fluorescent fusion. SecDF is part of the normal proteome and is a component of the SecYEG/SecDFYajC (YrbF in B. subtilis) protein secretion complex (Dalbey \& Chen, 2004). Thus, it does not appear that size is the criterion for temporary absence of certain proteins at mid-cell during cell division. Nevertheless, it was observed that septa containing GFPSecDF were visible before the AtpA-CFP fusion (see arrows, Fig. 2F), which may indicate that although both complexes are large, the ATP synthase complex diffuses more slowly than SecYEG/SecDFYajC.

We also investigated the possibility that CRs may be due to some aspect of DNA metabolism, as DNA replication has also been shown to occur around the mid-cell region. However, in strains depleted of FtsZ, in which cells became filamentous but underwent normal DNA replication and segregation, CR frequency rapidly dropped to zero, indicating that CRs were dependent in some way on cell division.

Quantitative analysis showed that CRs only occurred at the early stages of the division cycle, corresponding approximately to the time at which FtsZ rings first assemble. It had seemed possible that the physical crowding by divisionspecific proteins could be responsible for a passive exclusion of other proteins from the mid-cell. Two lines of evidence suggest this is not the case. Firstly, during the later stages of cell division when the level of division proteins is highest at mid-cell, CRs are not observed. They are only seen at the early stages of division when the mature divisome is still being assembled. Secondly, CRs were not observed with all integral membrane proteins suggesting they may represent a specific response by a subset of proteins.

It was also possible that the lipid composition of the membrane plays a role in CR formation, and there does appear to be a connection between local changes in lipid composition and cell division (Mileykovskaya \& Dowhan, 2005). A number of studies in both E. coli and B. subtilis using membrane- and CL-specific dyes indicate that midcell and polar regions have a different staining profile to cell-cylinder membrane, and are particularly rich in CL and PE (Fishov \& Woldringh, 1999; Mileykovskaya \& Dowhan, 2000; Kawai et al., 2004; Nishibori et al., 2005). There is some evidence that ATP synthase is associated with PG domains (Ksenzenko \& Brusilow, 1993), which are less abundant at sites of cell division. It could be that due to interaction with different subsets of lipids/lipid domains, some proteins/complexes are more mobile than others in the membrane and can diffuse into newly synthesized membranes before others (e.g. p16.7 and SecDF). Also, the enzymes responsible for synthesis of PE and CL localize to the division septum, where lipid synthesis is thought to occur (Nishibori et al., 2005), and this may inhibit the rate of diffusion of proteins embedded within PG-rich domains into the septal regions.

So, are CRs due to active exclusion or passive diffusion? The former is unlikely, as if that was the case, we would have expected to see them clearly in every cell early during 
the division cycle, whereas they were only seen in about $40 \%$ of cells at the early stages of cell division. It seems more likely that CRs are due to a slower rate of diffusion of certain complexes into newly synthesized membrane at the onset of cell division. The juxtaposition of membrane domains to centre cell at the onset of division could affect how easily detected a CR is, hence they are only seen in about $40 \%$ of cells early in the division cycle. Nevertheless, CRs could be indicative of local changes in the membrane composition at mid-cell, which are important in the formation of an active divisome that may be important in targeting division proteins to that region of the cell.

\section{ACKNOWLEDGEMENTS}

M.P. was supported by funding from the Bundesministerium für Bildung und Forschung (Germany). We thank E. Harry for providing anti-FtsZ antiserum. This work was supported by funding from the Australian Research Council grant DP0449482 and NHMRC project grant 455597.

\section{REFERENCES}

Anagnostopoulos, C. \& Spizizen, J. (1961). Requirements for transformation in Bacillus subtilis. J Bacteriol 81, 741-746.

Beall, B. \& Lutkenhaus, J. (1991). FtsZ in Bacillus subtilis is required for vegetative septation and for asymmetric septation during sporulation. Genes Dev 5, 447-455.

Binenbaum, Z., Klyman, E. \& Fishov, I. (1999). Division-associated changes in membrane viscosity of Escherichia coli. Biochimie 81, 921-929.

Dalbey, R. E. \& Chen, M. (2004). Sec-translocase mediated membrane protein biogenesis. Biochim Biophys Acta 1694, 37-53.

Davies, K. M. \& Lewis, P. J. (2003). Localization of rRNA synthesis in Bacillus subtilis: characterization of loci involved in transcription focus formation. J Bacteriol 185, 2346-2353.

Davies, K. M., Dedman, A., van Horck, S. \& Lewis, P. J. (2005). The NusA: RNA polymerase ratio is increased at sites of rRNA synthesis in Bacillus subtilis. Mol Microbiol 57, 366-379.

de Mendoza, D., Schujman, G. E. \& Aguilar, P. S. (2002). Biosynthesis and function of membrane lipids. In Bacillus subtilis and its Closest Relatives: from Genes to Cells, pp. 43-55. Edited by A. L. Sonenshine, J. A. Hoch \& R. Losick. Washington, DC: ASM Press.

Errington, J., Daniel, R. A. \& Scheffers, D.-J. (2003). Cytokinesis in bacteria. Microbiol Mol Biol Rev 67, 52-65.

Feucht, A. \& Lewis, P. J. (2001). Improved plasmid vectors for the production of multiple fluorescent protein fusions in Bacillus subtilis. Gene 264, 289-297.

Fishov, I. \& Woldringh, C. L. (1999). Visualization of membrane domains in Escherichia coli. Mol Microbiol 32, 1166-1172.

Gilson, P. R. \& Beech, P. L. (2001). Cell division protein FtsZ: running rings around bacteria, chloroplasts and mitochondria. Res Microbiol 152, 3-10.

Hamoen, L. W., Meile, J. C., de Jong, W., Noirot, P. \& Errington, J. (2006). SepF, a novel FtsZ-interacting protein required for a late step in cell division. Mol Microbiol 59, 989-999.
Harry, E., Monahan, L. \& Thompson, L. (2006). Bacterial cell division: the mechanism and its precision. Int Rev Cytol 253, 27-94.

Jenkinson, H. F. (1983). Altered arrangement of proteins in the spore coat of a germination mutant of Bacillus subtilis. J Gen Microbiol 129, 1945-1958.

Johnson, A. S., van Horck, S. \& Lewis, P. J. (2004). Dynamic localization of membrane proteins in Bacillus subtilis. Microbiology 150, 2815-2824.

Katis, V. L., Harry, E. J. \& Wake, R. G. (1997). The Bacillus subtilis division protein DivIC is a highly abundant membrane-bound protein that localizes to the division site. Mol Microbiol 26, 1047-1055.

Kawai, F., Shoda, M., Harashima, R., Sadaie, Y., Hara, H. \& Matsumoto, K. (2004). Cardiolipin domains in Bacillus subtilis Marburg strains. J Bacteriol 186, 1475-1483.

Ksenzenko, S. M. \& Brusilow, W. S. A. (1993). Protein--lipid interactions of the proteolipid c subunit of the Escherichia coli protontranslocating adenosinetriphosphatase. Arch Biochem Biophys 305, 78-83.

Lemon, K. P. \& Grossman, A. D. (1998). Localization of bacterial DNA polymerase: evidence for a factory model of replication. Science 282, 1516-1519.

Lewis, P. J. \& Marston, A. L. (1999). GFP vectors for controlled expression and dual labelling of protein fusions in Bacillus subtilis. Gene 227, 101-109.

Lewis, P. J., Thaker, S. D. \& Errington, J. (2000). Compartmentalization of transcription and translation in Bacillus subtilis. EMBO J 19, 710-718.

Matsumoto, K., Kusaka, J., Nishibori, A. \& Hara, H. (2006). Lipid domains in bacterial membranes. Mol Microbiol 61, 1110-1117.

Meijer, W. J., Serna-Rico, A. \& Salas, M. (2001). Characterization of the bacteriophage phi29-encoded protein p16.7: a membrane protein involved in phage DNA replication. Mol Microbiol 39, 731-746.

Migocki, M. D., Lewis, P. J., Wake, R. G. \& Harry, E. J. (2004). The midcell replication factory in Bacillus subtilis is highly mobile: implications for coordinating chromosome replication with other cell cycle events. Mol Microbiol 54, 452-463.

Mileykovskaya, E. \& Dowhan, W. (2000). Visualization of phospholipid domains in Escherichia coli by using the cardiolipin-specific fluorescent dye 10-N-nonyl acridine orange. J Bacteriol 182, 1172-1175.

Mileykovskaya, E. \& Dowhan, W. (2005). Role of membrane lipids in bacterial division-site selection. Curr Opin Microbiol 8, 135-142.

Nishibori, A., Kusaka, J., Hara, H., Umeda, M. \& Matsumoto, K. (2005). Phosphatidylethanolamine domains and localization of phospholipids synthases in Bacillus subtilis membranes. J Bacteriol 187, 2163-2174.

Rothfield, L., Taghalout, A. \& Shih, Y.-L. (2005). Spatial control of bacterial division-site placement. Nat Rev Microbiol 3, 959-968.

Sharpe, M. E., Hauser, P. M., Sharpe, R. G. \& Errington, J. (1998). Bacillus subtilis cell cycle as studied by fluorescence microscopy: constancy of cell length at initiation of DNA replication and evidence for active nucleoid partitioning. J Bacteriol 180, 547-555.

Wu, L. J. \& Errington, J. (2004). Coordination of cell division and chromosome segregation by a nucleoid occlusion protein in Bacillus subtilis. Cell 117, 915-925.

Wu, L. J., Lewis, P. J., Allmansberger, R., Hauser, P. M. \& Errington, J. (1995). A conjugation-like mechanism for prespore chromosome partitioning during sporulation in Bacillus subtilis. Genes Dev 9, 1316-1326.

Edited by: B. Margolin 